Case

Report

\title{
A Spontaneous Abdominal Aortic Pseudoaneurysm Treated with N-butyl Cyanoacrylate and Coil Embolization: A Case Report
}

\author{
Chan Sun Kim, MD, ${ }^{1}$ Young Ho Choi, MD, ${ }^{2}$ Young Ho So, MD, ${ }^{2}$ and Jae-Sung Choi, $\mathrm{MD}^{3}$
}

\begin{abstract}
Pseudoaneurysms are vascular spaces vulnerable to pressure, and expansion or rupture of these spaces may occur during embolization. Here, we describe the case of a transcatheter embolization of a spontaneous aortic pseudoaneurysm, which showed gradual expansion during n-butyl cyanoacrylate embolization. This pseudoaneurysm was successfully embolized with an adjuvant coil.
\end{abstract}

Keywords: aorta, pseudoaneurysm, transcatheter, embolization

\section{Introduction}

A spontaneous abdominal aortic pseudoaneurysm is not common, and surgical repair is the standard of treatment. However, endovascular management has recently emerged as an alternative due to advanced techniques and devices. Previous studies reported lower morbidity and mortality rates with the endovascular treatment of pseudoaneurysms than with surgery. ${ }^{1,2)}$ However, it should be kept in mind that expansion or rupture of a pseudoaneurysm could happen during endovascular procedures. Here, we describe a case of abdominal aortic pseudoaneurysm showing intraprocedural expansion of the pseudoaneurysmal sac during a transcatheter embolization.

\footnotetext{
${ }^{1}$ Department of Radiology, Seoul National University Hospital, Seoul, Korea

${ }^{2}$ Department of Radiology, Seoul Metropolitan Government Seoul National University Boramae Medical Center, Seoul, Korea

${ }^{3}$ Department of Thoracic and Cardiovascular Surgery, Seoul Metropolitan Government Seoul National University Boramae Medical Center, Seoul, Korea
}

Received: February 20, 2017; Accepted: March 23, 2017

Corresponding author: Young Ho Choi, MD. Department of Radiology, Seoul Metropolitan Government Seoul National University Boramae Medical Center, 20 Boramae-ro 5-gil, Dongjak-gu, Seoul 156-707, Korea

Email: cyho50168@naver.com

(C)2018 The Editorial Committee of Annals of Thoracic and Cardiovascular Surgery. All rights reserved.

\section{Case Report}

A 35-year-old man with a history of moyamoya disease, intracranial hemorrhage, epilepsy, chronic pancreatitis, aspiration pneumonia, and end-stage renal disease (on hemodialysis) was admitted to the neurosurgery department following a seizure. A computed tomography (CT) scan of the brain showed an intracranial hemorrhage in the right frontoparietal lobe. Therefore, the patient underwent an emergent craniotomy and evacuation of the hematoma.

After 4 months, the patient developed melena, and although his blood pressure and pulse rate were stable, his hemoglobin level was decreased from 8.0 to $5.8 \mathrm{~g} / \mathrm{dL}$. An esophagogastroscopy revealed hemorrhagic gastritis and gastric fundal varices. Since there was no evidence of liver cirrhosis or other manifestations of portal hypertension, an abdominal CT was performed to elucidate the cause of the gastric varices. The abdominal CT revealed a $14 \times 10 \mathrm{~mm}$ pseudoaneurysm in the aortocaval region of the infrarenal aorta (Fig. 1A). About 17 months earlier, he had undergone an abdominal CT scan for the evaluation of pancreatitis, and the aorta had been unremarkable. This patient had no previous history of abdominal surgery or trauma over the previous 2 years, but given his complicated morbidities, an open surgical repair was considered to carry a high potential for mortality. Therefore, a transcatheter embolization of the pseudoaneurysm was selected, considering the narrow neck and challenging anatomy for a percutaneous approach. 

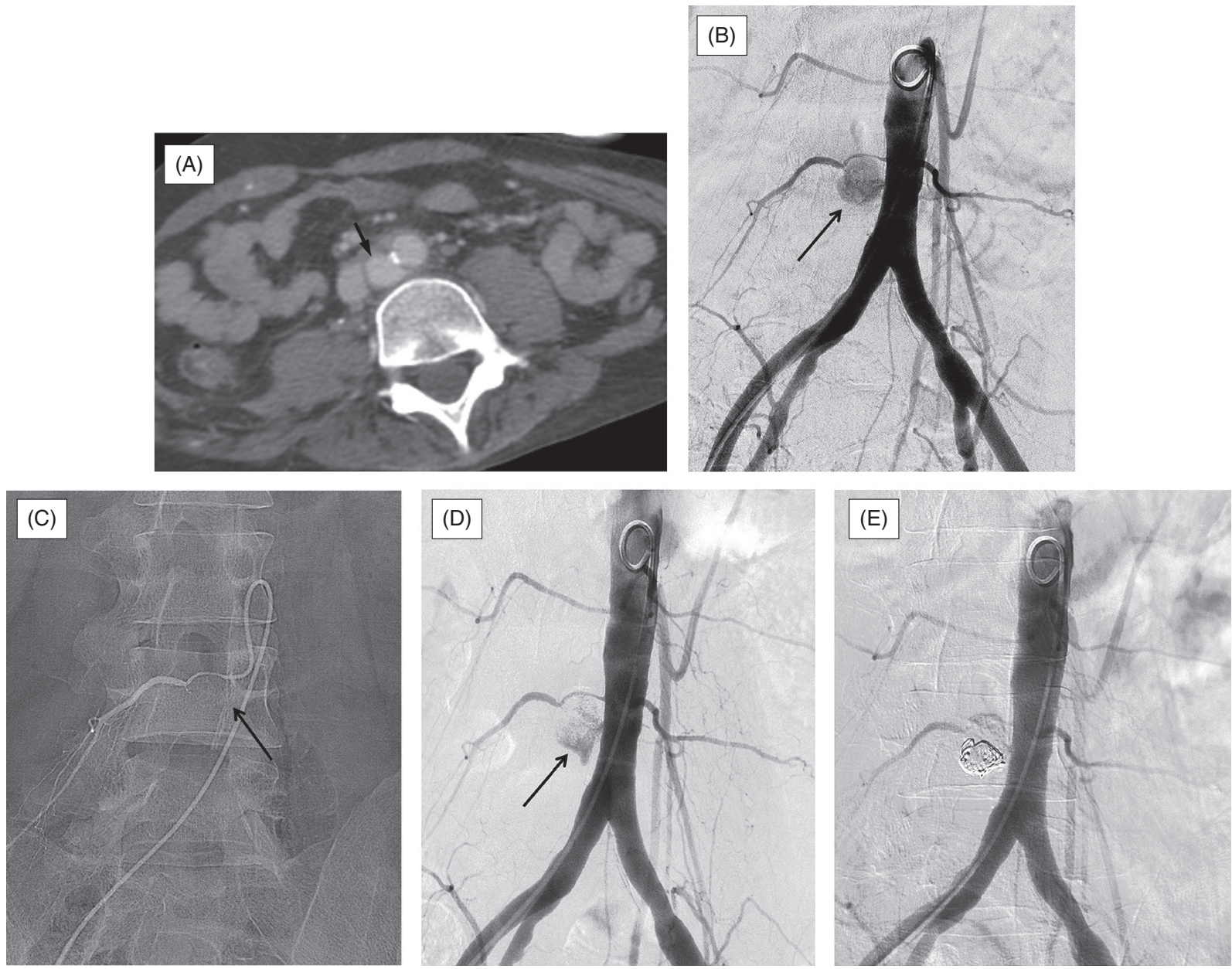

Fig. 1 A 35-year-old male with an abdominal aortic pseudoaneurysm. (A) Abdominal CT scan showing a $14 \times 10 \mathrm{~mm}$, saccular pseudoaneurysm in the aortocaval region of the infrarenal aorta (arrow). (B) Digital subtraction angiography showing a pseudoaneurysm just below the 4th lumbar artery (arrow). (C) Selective 4th lumbar arteriography revealing no connection between the pseudoaneurysm and the 4th lumbar artery. A small defect of calcification is noted on the right aortic wall (arrow). (D) Angiography after n-butyl cyanoacrylate embolization showing an increased sac size and a changed sac shape with incomplete exclusion (arrow). (E) Angiography after adjuvant coil embolization showing complete elimination of the pseudoaneurysm.

A retrograde puncture of the right common femoral artery was performed, with placement of a pigtail catheter. A diagnostic aortogram revealed a saccular pseudoaneurysm just below the 4th lumbar artery (Fig. 1B). This was followed by a selective 4th lumbar arteriogram, which showed no connection between the pseudoaneurysm and the 4th lumbar artery. However, a fissure in the right infrarenal aortic wall was consistent with the orifice of the pseudoaneurysm (Fig. 1C), and a 5-Fr RH catheter (Cook, Bloomington, IN, USA) and $0.035^{\prime \prime}$ angled guidewire (Terumo, Somerset, NJ, USA) were used to select the orifice. After confirmation of the exact catheterization with the injection of contrast, an n-butyl cyanoacrylate and lipiodol mixture (1:1 ratio) was injected into the sac. During the injection, the sac expanded slightly and slowly, and the injection was stopped. An angiography was performed, and demonstrated incomplete elimination of the pseudoaneurysm, shape change of the sac, and increase in the sac size (Fig. 1D). For adjuvant embolization, eight interlock coils (Boston Scientific, Natick, MA, USA) were then deployed in the pseudoaneurysmal sac. Upon completion, an angiogram revealed successful elimination of the pseudoaneurysm (Fig. 1E) without any further change in the sac shape or size.

Due to complicated comorbidities, the patient was not discharged until 2 months after the procedure. However, the patient experienced no adverse events during 
his hospital stay. The pseudoaneurysm was confirmed to be completely embolized in CT performed 10 months later.

\section{Discussion}

The causes of pseudoaneurysms are diverse, and include trauma, ${ }^{3)}$ infection, ${ }^{4)}$ inflammation, ${ }^{5)}$ and various iatrogenic events (e.g., surgery, catheterization, and percutaneous procedures). However, aortic pseudoaneurysms are most frequently associated with postoperative anastomotic complications. ${ }^{1)}$ In our case, the etiology of the pseudoaneurysm was unclear. Although the patient had a history of chronic pancreatitis, the affected infrarenal aorta was far from the pancreas. Moreover, the CT scan showed no demonstrable surrounding soft tissue infiltration or abnormal fluid collection that could suggest the possibility of infection. Young age and his complicated medical history (end-stage renal disease without diabetes mellitus, gastric varix bleeding without liver cirrhosis, and spontaneous intracranial hemorrhage) might show the possibility that he had any of the systemic vascular disorders.

In contrast to true aneurysms, pseudoaneurysms are surrounded by an injured arterial wall or single fibrous tissue, and they must be treated without delay, whatever their size. Traditionally, the treatment has been surgery, but in recent years, minimally invasive interventions, including percutaneous, transcatheter management, and endovascular stent grafts, have been developed as alternatives to surgery. However, these options should be tailored to the location, size of the neck, rupture risk, and patient comorbidities. If a patient has any of the systemic vascular diseases, surgery and stent-graft placement had better be avoided in that other vascular problems including another pseudoaneurysm and stent-graft function failure due to tissue involvement adjacent to the primary focus and aortic dilatation could happen.

Percutaneous management is appropriate when the pseudoaneurysm is endoluminally inaccessible, and is safe to puncture. Injections of thrombin, as well as coils or n-butyl cyanoacrylate, can also be used. A percutaneous thrombin injection under ultrasonographic guidance has been widely accepted as an effective treatment for superficially located pseudoaneurysms (e.g., a postcatheterization pseudoaneurysm). Since a distal thromboembolism may occur as one complication, a thrombin injection should be monitored closely with ultrasonography. Some authors have described the use of endovascular balloon occlusion of the pseudoaneurysmal neck to prevent distal thromboembolisms, but this approach makes the procedure more invasive, and increases the risk of puncturerelated complications. ${ }^{6}$ For example, Lin et al. ${ }^{7}$ reported a case with a transcatheter intra-arterial thrombin injection used to treat an ascending aortic pseudoaneurysm, which resulted in a disastrous thromboembolism in the innominate artery and a transient ischemic attack. Therefore, the transcatheter use of thrombin should be avoided, especially in a wide-neck aneurysm. Furthermore, the use of thrombin is commonly associated with allergic reactions, such as urticaria and anaphylaxis. ${ }^{8)}$ Considering these points, we chose n-butyl cyanoacrylate as an embolic agent to treat the pseudoaneurysm, instead of thrombin. However, bigger amount of n-butyl cyanoacrylate mixed with lipiodol, compared to thrombin liquid, was required to fill up the pseudoaneurysm sac. It caused internal pressure increase in the sac under the small neck of the pseudoaneurysm, which resulted in increase in the sac size and shape change of the sac in addition to incomplete embolization. Pseudoaneurysm wall is not real vascular wall and formation of the sac is achieved with a help of tissue surrounding an extravasated or leaked vascular pool. It means pseudoaneurysm wall could be fragile like in this case. The outer longitudinal diameter of the sac in CT scan before the embolization was about $20 \mathrm{~mm}$, and that in CT scan 10 months after the embolization was about $23 \mathrm{~mm}$. If the pseudoaneurysm bursts and needs an emergency surgery, it is much more invasive than scheduled surgery. For this reason, the worst scenario should be considered along with secondary management plan. Thrombin might be the better choice than n-butyl cyanoacrylate in that the amount of thrombin liquid used in embolization must be much smaller than n-butyl cyanoacrylate mixed with lipiodol.

The transcatheter embolization of a pseudoaneurysm can be performed with coils, vascular occluder devices, and n-butyl cyanoacrylate. In cases of aortic pseudoaneurysms, the neck or pseudoaneurysm itself is the target of embolization because the pseudoaneurysm must be excluded from the circulation while preserving the aorta. Hussain et al. ${ }^{9)}$ have reported six cases of aortic pseudoaneurysms treated with Amplatzer occluder devices. In their study, there were four cases with successful embolizations and two cases with failed embolizations due to wide necks. Amplatzer occluder devices are available in different sizes; thus, unlike coils, they can be used with pseudoaneurysms with relatively large necks. Overall, the size of the neck must be accurately assessed before using an occluder device, which should 
be avoided if such an assessment is impossible or if the neck is too broad.

When using coils and n-butyl cyanoacrylate, the target of embolization is the pseudoaneurysmal sac itself, and narrow-neck pseudoaneurysms can be embolized with catheter-directed delivery of these embolization materials. The use of a low-profile microcatheter with good flexibility is the major advantage of this procedure; however, if the neck is wide, there is a major risk of distal migration of the embolization materials, and stent- or balloon-assisted remodeling is required to prevent this migration. There is also a risk of expansion or rupture of the sac during the embolization, due to the weak, single fibrous layer surrounding the pseudoaneurysmal space.

Stent-graft placement is the preferred endovascular option to treat abdominal aortic pseudoaneurysms. It requires a higher profile and stiffer delivery system, and must be placed in larger arteries to prevent thrombosis. However, these factors are not an issue when deploying stents in the abdominal aorta. The placement of the stent graft across the neck can eliminate wide-neck pseudoaneurysms, which cannot be readily achieved with transcatheter embolization. In cases of visceral artery involvement, hybrid techniques, such as a transposition or bypass, can be considered.4) It is well known that stent-graft placement is contraindicated in mycotic aneurysms and pseudoaneurysms. However, a recent multicenter study ${ }^{10)}$ showed that a stent-graft insertion with prolonged antibiotic therapy in mycotic aortic aneurysms was a durable treatment option, with favorable short-term and long-term outcomes. ${ }^{4)}$

\section{Conclusion}

There are various endovascular approaches to the management of aortic pseudoaneurysms, and the determination of the optimal treatment method should be based on anatomical and morphological considerations. However, given the pathophysiology of pseudoaneurysms, intraprocedural expansion or rupture may occur. When performing a transcatheter embolization of the pseudoaneurysmal sac, secondary alternatives, such as embolization with different materials and stent-graft placement, should be prepared and planned for in advance.

\section{Disclosure Statement}

We have no conflicts of interest.

\section{References}

1) Quevedo HC, Santiago-Trinidad R, Castellanos J, et al. Systematic review of interventions to repair ascending aortic pseudoaneurysms. Ochsner J 2014; 14: 576-85.

2) Saad NE, Saad WE, Davies MG, et al. Pseudoaneurysms and the role of minimally invasive techniques in their management. Radiographics 2005; 25: S173-89.

3) Tucker S, Rowe VL, Rao R, et al. Treatment options for traumatic pseudoaneurysms of the paravisceral abdominal aorta. Ann Vasc Surg 2005; 19: 613-8.

4) Soule M, Javerliat I, Rouanet A, et al. Visceral debranching and aortic endoprosthesis for a suspected mycotic pseudoaneurysm of the abdominal aorta involving visceral arteries. Ann Vasc Surg 2010; 24: 825.e13-6.

5) Corona M, Boatta E, Lucatelli P, et al. Aortic pseudoaneurysm rupture secondary to pancreatitis: an endovascular approach. Diagn Interv Imaging 2015; 96: 515-7.

6) Lennox AF, Delis KT, Szendro G, et al. Duplexguided thrombin injection for iatrogenic femoral artery pseudoaneurysm is effective even in anticoagulated patients. Br J Surg 2000; 87: 796-801.

7) Lin PH, Bush RL, Tong FC, et al. Intra-arterial thrombin injection of an ascending aortic pseudoaneurysm complicated by transient ischemic attack and rescued with systemic abciximab. J Vasc Surg 2001; 34: 939-42.

8) La Perna L, Olin JW, Goines D, et al. Ultrasoundguided thrombin injection for the treatment of postcatheterization pseudoaneurysms. Circulation 2000; 102: 2391-5.

9) Hussain J, Strumpf R, Wheatley G, et al. Percutaneous closure of aortic pseudoaneurysm by Amplatzer occluder device-case series of six patients. Catheter Cardiovasc Interv 2009; 73: 521-9.

10) Sörelius K, Mani K, Björck M, et al. Endovascular treatment of mycotic aortic aneurysms: a European multicenter study. Circulation 2014; 130: 2136-42. 